\title{
Paravertebral catheters for rib fractures: a case series
}

\author{
Dr H. Doherty ${ }^{1}$, Dr S. Cope², Dr A. Mann².
}

${ }^{1}$ Northern School of Anaesthesia and Intensive Care Medicine, Anaesthetics, Newcastle upon Tyne, United Kingdom. ${ }^{2}$ City Hospitals Sunderland NHS Foundation Trust, Anaesthetics, Sunderland, United Kingdom.

\section{Background}

Rib fractures are seen commonly in blunt thoracic trauma. ${ }^{1}$ Good analgesia is essential to allow adequate tidal volume and cough, aid chest physiotherapy, and reduce the risk of morbidity and mortality from respiratory complications. ${ }^{1,2}$ Multimodal analgesia should be initiated, $1,3,4$ and various regional analgesic options are available. Thoracic epidural is the gold standard when multimodal analgesia has failed ${ }^{1,4}$, potentially reducing the duration of mechanical ventilation requirement ${ }^{5}$ and incidence of respiratory complications. ${ }^{6}$ However, its side effect profile is a disadvantage. There is evidence that a paravertebral catheter (PC) can provide as effective analgesia with fewer side effects and complications than an epidural. ${ }^{7}$

\section{Aims}

A blunt thoracic trauma pathway was introduced in January 2017 to aid management of patients with blunt thoracic trauma at high risk of respiratory complications, who may benefit from regional analgesia. Patients should be referred to the block room anaesthetist for consideration for a PC. The aim of this project was to evaluate the analgesic benefit provided by PCs in this group of patients.

\section{Methods}

We kept a record of patients who had a PC inserted for one year after introduction of the blunt thoracic trauma pathway. After medical assessment to rule out contraindications and informed consent, patients had a PC inserted in the block room under ultrasound guidance and full asepsis, using a Pajunk Sonolong Curl Echo catheter kit. A bolus dose of $0.25 \%$ levobupivacaine, followed by infusion of $0.2 \%$ Ropivacaine were given at a dose appropriate to the patient. Patients were managed on the ward or critical care as appropriate, with daily acute pain team reviews. The project was approved by the trust's Clinical Governance department who did not require ethics approval. Data was collected for fifteen patients and compared to data for nine patients admitted in the year prior to introduction of the pathway, who met the criteria for PC but did not have one. Wilcoxon signed-rank test was used to determine statistical significance for the comparison of pre-insertion, immediate post-insertion, and 24 hours pain scores. Mann Whitney U test was used to compare 24-hour pain scores between the two groups.

\section{Results}

Pre-insertion pain score [median (IQR)]; documented in 93\% patients; was 7 (2.5). Immediate post-insertion pain score [median (IQR)]; documented in $93 \%$ patients was $0(3.5)$. (Fig. 1$)$. 9 patients $(60 \%)$ had a pain score of zero immediately post-insertion and 12 patients $(80 \%)$ had a pain score of 5 or less. The difference between pre-insertion and immediate post-insertion pain scores was statistically significant $(p=0.002)$.

24 hour post-insertion pain score [median (IQR)]; documented in 93\% patients; was $3(7.25)$. (Fig. 1). 5 patients $(33 \%)$ had a pain score of zero at 24 hours and 10 patients $(67 \%)$ had a pain score of 5 or less. The difference between pre-insertion and 24 hour pain scores was statistically significant $(p=0.008)$.

9 patients $(60 \%)$ had a sustained reduction in pain score at 24 hours. One patient had an initially poor response, but with a reduction in pain score to 3 at 24 hours. 3 patients had an initial fall in pain score, but an increase at 24 hours (Fig. 2). 2 patients were excluded from the graph due to insufficient data.

The duration of catheter [mean (range)] was 67.9 (24-144) hours. In the 9 patients who did not have a PC, pain score on admission [median $(\mathrm{IQR})$ ] was 8 (1.5). Pain score 12 hours after admission [median (IQR)]; documented in $(89 \%)$ patients; was 9 (2). Pain score 24 hours after admission; documented in (89\%) patients was 7.5 (5). (Fig. 3). A statistically significant difference in 24 hour pain scores was observed between the two groups $(p=0.01)$.

Figure 3: 24 hour pain scores for those with a paravertebral catheter compared to those without

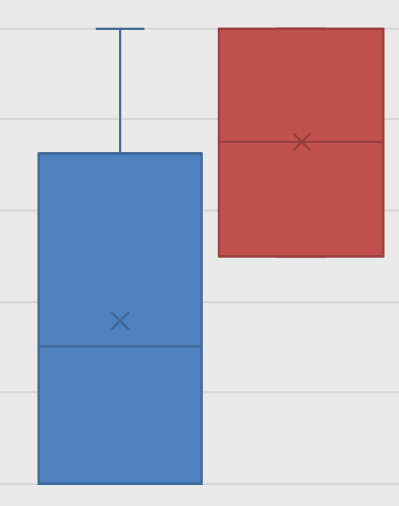

Figure 1: Pre- and post-insertion pain scores

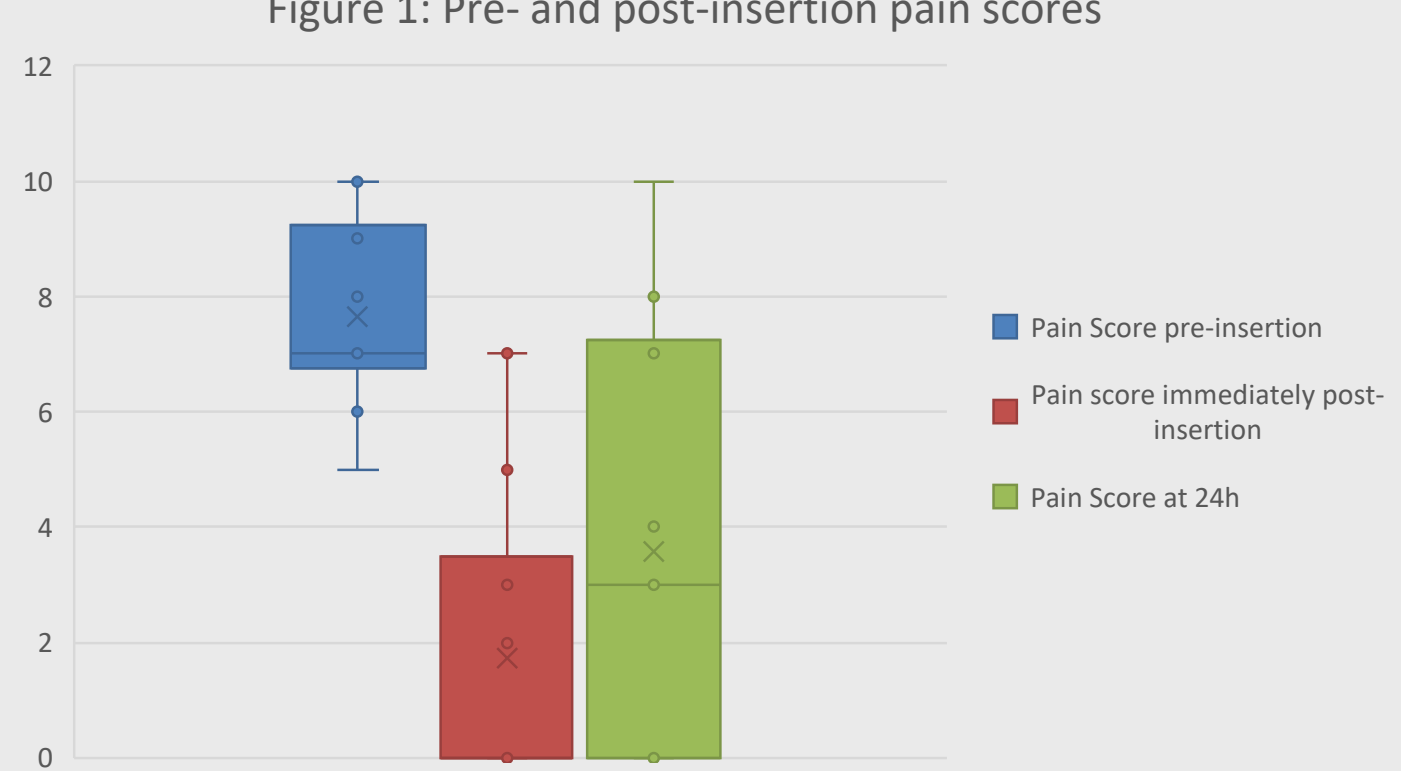

Figure 2: Pain Scores over time for patients for whom all data available

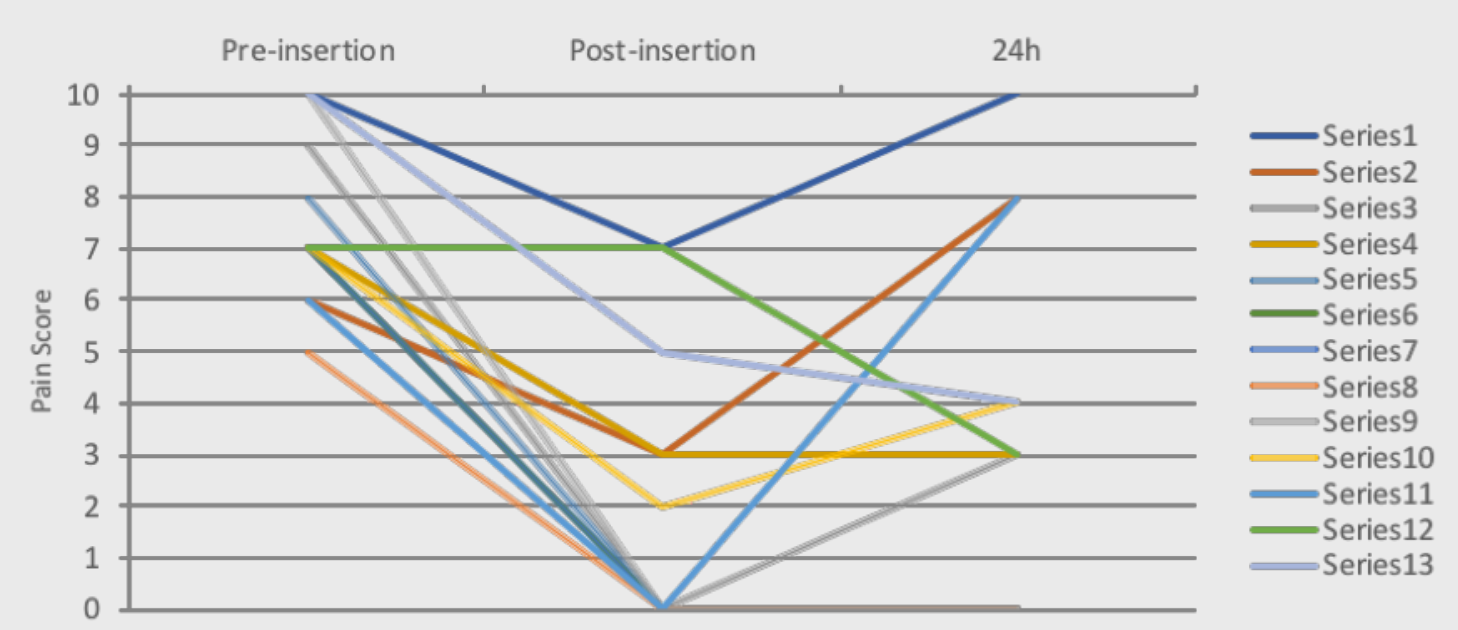

\section{Conclusion}

15 patients receiving PC local anaesthetic infusion were compared to 9 similar patients who received oral analgesia. Statistically significant reductions in pain scores were seen after PC insertion and, although the number of patients is too small to observe a difference in the rate of respiratory complications, we also observed significantly lower pain scores at 24 hours in the group of patients receiving paravertebral analgesia compared with those patients receiving oral analgesia.

\section{Discussion}

There are many proposed alternative regional anaesthetic techniques to the PC. Such include the serratus plane block, intrapleural and intercostal block. ${ }^{1}$ The Erector Spinae Plane (ESP) block is gaining popularity as an alternative to PCs due to the relative ease of insertion and lower complication rate, with comparable clinical outcome ${ }^{8}$ and our block room team are keen to develop this technique. We plan to collect the same data for patients receiving ESP catheters and compare it with the data presented here.

References:

References:

2.Pressley CM., Fry WR., Philp AS., Berry SD., Smith RS. Predicting outcome of patients with chest wall injury. The American Journal of Surgery 2012; 204(6): 910-13

3.Witt CE, Bulger EM. Comprehensive approach to the management of the patient with multiple rib fractures: a review and introduction of a bundled rib fracture management protocol. Trauma Surgery \& Acute Care Open 2017;2: 1-7

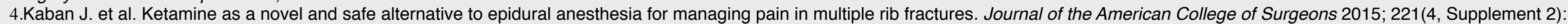
e143

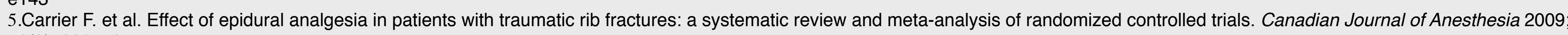
56(3): 230-42

6.Jarvis A.M. et al. Comparison of epidural versus parenteral analgesia for traumatic rib fractures: A meta-analysis. OPUS 12 Scientist 2009; $3(3)$ : 50-57

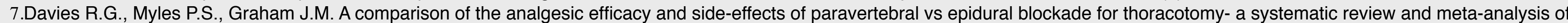

randomized trials. British Journal of Anaesthesia 2006; 96(4): 418-426 